\title{
Application of fluorescence imaging in lettuce phenotyping for drought stress tolerance
}

\author{
Marek ŽIVČÁK - Marek KOVÁR - Oksana SYTAR - Marián BRESTIČ \\ Department of Plant Physiology, Slovak University of Agriculture in Nitra, Tr. A. Hlinku 2, Nitra, 94976, \\ Slovakia; E-mail: marek.zivcak@uniag.sk
}

Keywords: plant phenotyping, lettuce, drought, fluorescence imaging

\section{Introduction}

Phenotyping of genetic resources is currently a "hot topic" in crop research, with a high focus put on stress tolerance studies. Chlorophyll fluorescence imaging (CFI) represents an important technique involved in numerous phenotyping platforms. CFI is expected to serve as a measure of photosynthetic functions, especially in studies of genotype $\times$ environment interactions (Harbinson et al., 2012). In our study, we tested opportunity to apply CFI to recognize drought sensitivity in lettuce genotypes.

\section{Materials and methods}

Eight genetically distinct parental lines (PL-1 to PL-8) of cultivated lettuce (Lactuca sativa L.) and one drought resistant wild lettuce (Lactuca serriola L.; LS) were cultivated in a growth chamber under limited/non-limited water supply $(20 \% / 70 \%$ of available water capacity). At the end of the experiment, plants were exposed to severe drought stress by withholding of irrigation for 3 more days. In parallel with other phenotyping methods applied in the experiment, CFI was recorded by IMAGING-PAM device (Walz, Germany) regularly in light exposed plants at the ambient light intensity.

\section{Results and discussion}

Table 1: Mean values of plant dry mass and fluorescence parameters measured in lettuce parental lines

\begin{tabular}{ccccccc}
\hline \multirow{2}{*}{ Genotype } & \multicolumn{2}{c}{ Plant dry mass, g/plant } & \multicolumn{2}{c}{ Electron Transport Rate, ETR } & \multicolumn{2}{c}{ Fluorescence value, Fs' } \\
\cline { 2 - 7 } & WW & DS & WW & DS & WW & DS \\
\hline PL-1 & 2.01 & 1.41 & 36.4 & 27.4 & 0.246 & 0.116 \\
PL-2 & 2.58 & 1.35 & 51.5 & 30.9 & 0.168 & 0.069 \\
PL-3 & 1.81 & 1.21 & 45.7 & 27.4 & 0.227 & 0.092 \\
PL-4 & 2.81 & 1.41 & 51.0 & 29.9 & 0.224 & 0.079 \\
PL-5 & 1.99 & 1.56 & 53.9 & 35.9 & 0.100 & 0.043 \\
PL-6 & 1.95 & 1.19 & 52.9 & 33.0 & 0.163 & 0.068 \\
PL-7 & 1.75 & 1.11 & 59.0 & 33.0 & 0.177 & 0.062 \\
PL-8 & 2.41 & 1.41 & 49.1 & 23.6 & 0.238 & 0.065 \\
LS & 1.62 & 1.17 & 59.2 & 32.8 & 0.191 & 0.061 \\
\hline
\end{tabular}

WW-well watered; DS-drought stress

Depending on genotypes, total dry mass in drought stressed plants decreased by $20-50 \%$ compared to control; the relative plant dry mass decrease (DMD) was used as a measure of drought sensitivity of genotypes. CFI analyses have shown a significant decrease in the apparent electron transport rate, ETR, in all genotypes (having the same trend as the efficient quantum yield, $\left.\Phi_{\text {PSII }}\right)$. We observed also a significant decrease of steady-state fluorescence intensity (Fs), both in moderate and severe drought. Fs decrease can be 
explained by activation of energy-dissipating processes (non-photochemical quenching) and/or structural changes in the leaves. The correlation analyses indicated only o low correlation between DMD and ETR in severe drought stress $(r=0.45)$, but the relatively good correlation between DMD and Fs' observed in severe drought $(\mathrm{r}=0.94)$. The better correlation in severe drought can be well explained by the fact that all plants were functionally in a very similar status (stomata closed, photosynthesis stopped), and the differences in Fs' were dominantly due to structural changes (Kalaji et al., 2017).
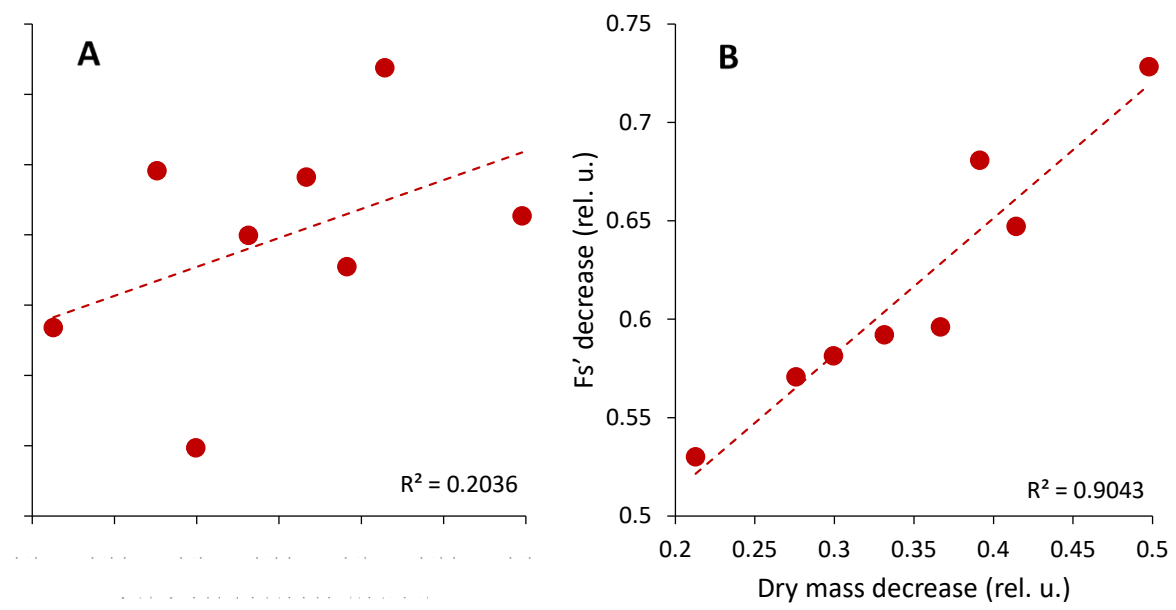

Figure 1: Relationship between dry mass decrease (as a measure of drought impairment in lettuce genotypes) and electron transport rate records during period of severe drought stress $(A)$ and the relative decrease of the steady state fluorescence ( $\left.F s^{\prime}\right)$ during period of severe drought stress $(B)$.

\section{Conclusions}

Based on the results, the steady-state fluorescence signal (Fs') reflected well the effects of water deficit on photosynthetic apparatus in phenotyping for drought tolerance in lettuce. In contrary, parameter ETR often referred as the most useful parameter for phenotyping, was not sensitive enough. This is an important information emphasizing the need of testing the techniques in individual crops and different stress scenarios.

\section{Acknowledgement}

Supported by the national grants APVV-15-0721 and VEGA-1-0831-17.

\section{References}

Harbinson, J., Prinzenberg, A. E., Kruijer, W., Aarts, M. G. (2012): High throughput screening with chlorophyll fluorescence imaging and its use in crop improvement. Current Opinion in Biotechnology, 23: 2. 221-226. DOI: http://dx.doi.org/10.1016/j.copbio.2011.10.006

Kalaji MH, Goltsev V, Zuk-Golaszewska B, Zivcak M, Brestic M (2017): Chlorophyll fluorescence: understanding crop performance-basics and applications. CRC Press, Taylor and Francis Group, Boca Raton, New York, London 